\title{
Clinico-epidemiological Profile of Onychomycosis Attending in a Tertiary Care Hospital
}

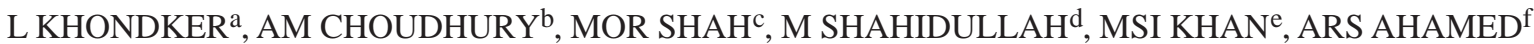

\section{Summary:}

A cross sectional study, conducted in the department of Dermatology and Venereology, Bangabandhu Sheikh Mujib Medical University (BSMMU) for duration of January 2009 to December 2010. Hundred twenty patients with onychomycosis were selected by purposive type of nonprobability sampling technique. Majority of the patients $61(150.8 \%)$ were in the age group of 21-30 years old. Mean age of the patients were $32.8 \pm 14$ years and most of the patients were house wives $36(30.0 \%)$. Disfigurement 117(97.5\%) and discomfort 89(74.2\%) were more common chief complaints of the patients. The mean duration of disease was 20.4 \pm 15.4 months and nail fold changes were associated with $37(30.8 \%)$ patients and more than a half 63(52.5\%) of the patients had history of wet works. Regarding the history of past illness, it was observed that previous onychmycosis found 26(21.7\%), nail trauma 26(21.7\%) and immune suppression 6(5.0\%). In endocrinopathies, hypothyroidism was observed in 1(0.8\%),

a. Dr. Lubna Khondker, MBBS, MPH, DDV, MCPS, FCPS (Dermatology and Venereology), Assistant professor, Dept of Dermatology and Venereology, Bangabandhu Sheikh Mujib Medical University, Shahbag, Dhaka.

b. Dr. Agha Masood Choudhury, MBBS, DDV, DD, MS. Professor and Chairman, Dept of Dermatology and Venereology, Bangabandhu Sheikh Mujib Medical University, Shahbag, Dhaka.

c. Col.(Dr) Md Obaidur Rahman Shah , MBBS, DDV, FCPS, Classified Specialist in Dermatology and Venereology, Combined Military Hospital (CMH), Dhaka Cantt, Dhaka.

d. Dr. Md Shahidullah, MBBS, DDV, MCPS, FCPS, FRCP, Professor and Head, Dept of Dermatology and Venereology, Shaheed Monsur Ali Medical College Hospital, Dhaka.

e. Major (Dr). Md Shirajul Islam Khan, MBBS, MCPS, DDV, Graded Specialist in Dermatology and Venereology, Combined Military Hospital (CMH), Dhaka Cantt, Dhaka.

f. Dr. Abu Reza Sayem Ahamed, MBBS, MCPS, DDV, Junior Consultant in Dermatology and Venereology Directorate General of Health, Mohakhali, Dhaka.

Address of Correspondence: Dr. Lubna Khondker, MBBS, DDV, MCPS, FCPS (Dermatology), Assistant Professor, Dept of Dermatology and Venereology, Bangabandhu Sheikh Mujib Medical University (BSMMU). Shahbag, Dhaka, Bangladesh. Mobile01552370429, E-mail: lubna_derma@yahoo.com.

Received: 06 June, 2011

Accepted: 22 February, 2012
Diabetes Mellitus 6(5.0\%) etc. Regarding the pattern of nail changes, thickening of nail plate 88(73.3\%), onycholysis 67(55.8\%), subungunal hyperkeratosis 61(50.8\%) were more common changes. Paronychia was observed in 34 (28.3\%) cases. In concomitant fungal infection, it was observed that T. manuum in $6(5.0 \%)$, T. pedis $3(2.5 \%)$, Interdigital intertrigo $2(1.7 \%)$ and T. cruris $1(0.8 \%)$. This was a study on a limited number of cases. Future studies must include economical support, then large sample size could be ensured and study finding would be more reliable. There is a great need of epidemiological studies also, with sufficient follow-up, systematic reviews and meta-analyses on this issue.

Key words: Onychomycosis, clinical profile, epidemiological profile.

(J Bangladesh Coll Phys Surg 2012; 30: 78-84)

Introduction:

Onychomycosis comprises all fungal infections affecting the nail apparatus, i.e., nail plate, nail bed, nail matrix, nail folds, cuticle and hyponychium. ${ }^{1}$ It accounts for upto $50 \%$ of all nail disorders and $30 \%$ of all superficial fungal infections of skin. ${ }^{2,3}$ The dermatophytes cause the great majority of onychomycosis. Trichophyton rubrum is responsible for approximately 71 percent of all cases and Trichophyton mentagrophytes add another 20 percent. Yeasts are the source of approximately 5 percent of onychomycosis, the majority of which is caused by candida albicans. The nondermatophyte moulds Acremonium, Aspergillus, Fusarium, Onychocola canadensis, Scopulariopsis brevicalis and Scytalidium dimidiatum account for approximately 4 percent of onychomycosis. ${ }^{1}$

Onychomycosis expresses itself in various forms and clinically the disease was classified as distal and lateral subungual onychomycosis(DLSO), superficial white onychomycosis(SWO), proximal subungual onychomycosis(PSO), and candidial onychomycosis. ${ }^{4}$ Patient may have combination of these various forms. Total dystrophic onychomycosis refers to most advanced form of any of the above. ${ }^{3,5}$ Nail changes in onychomycosis can occur in various forms, such as destruction of nail plate, roughening of nailplate, 
onycholysis, subungual hyperkeratosis, thickening and discoloration of nail plate (yellowish, brownish-yellow, whitish, blackish). ${ }^{6}$ Onychomycosis is common in Bangladesh, but no significant data was available in this country. Here an endeavor had been made to find out the clinico-epidemiological profile od onychomycosis.

\section{Materials and Methods:}

It was a cross sectional study, conducted in the department of Dermatology and Venereology, BSMMU for a duration of January 2009 to December 2010. Hundred twenty patients with onychomycosis, attending in outpatient department, were selected by purposive type of non-probability sampling technique, considering inclusion and exclusion criteria of patient selection.

\section{Inclusion criteria:}

i) Clinically diagnosed cases of onychomycosis having destruction of nail plate, onycholysis, subungual hyperkeratosis, discoloration \& thickening of nail plate alone or in combination.

ii) Patients of both sexes \& all ages.

iii) Patients who were given informed consent.

\section{Exclusion criteria:}

i) Not willing to participate.

ii) Patients who had received treatment either with topical and / or systemic antifungal agents for present nail condition within the last one month.

iii) Diagnosed cases of other dermatological diseases having nail changes eg. Psoriasis,

Lichen Planus, Eczema, PRP, Darier’s disease.

\section{Ethical consideration:}

i) All information of benefits and hazards was delivered to the patient.

ii) Patient was informed about the methodology, objective and purposes of the study.

iii) Patient's consent was taken without any influence.

iv) Information obtained from the patient was kept confidential.

All patients with onychomycosis attending in department of Dermatology and Venereology, BSMMU were examined. Then patients for this study were selected on the basis of history, clinical examination, inclusion and exclusion criteria.

\section{Clinical Assessment}

According to structured questionnaire, their particulars and history was taken. Demographic data, mainly age, sex, occupation and economic status were obtained from each patient. Patient complaints and duration of nail involvement were recorded. Specific data related to risk factors for onychomycosis (use of occlusive footwear, involvement of wet work, family history), predisposing factor such as previous onychomycosis, nail trauma, diabetes mellitus, concomitant fungal infections were noted.

After collection, data was checked for inadequacy, irrelevancy, and inconsistency. Irrelevant data was discarded. Analysis of data were performed by using SPSS (statistical package of social science) software.

\section{Results:}

This was a cross sectional study conducted in the department of Dermatology and Venereology, BSMMU, Dhaka. The main objective of the study was to find out the clinic-epidemiological profile of onychomycosis. A total of 120 patients of both men and women were enrolled in this study. Majority of the patients $61(150.8 \%)$ were in the age group of 2130 years old. Mean age of the patients were $32.8 \pm 14$ years. Male patients 64(53.3\%) were more than female $56(45.8 \%)$ in the study. Most of the patients were house wives 36 (30.0\%), followed by students (27.5\%), service holder (20.8\%) etc. More than a half (52.5\%) of the patients had involved in wet works, e.g. kitchen work, washing of cloths. Family history of any onychomycosis and sharing of common facilities present in $18.8 \%$ \& $15.0 \%$ of patient respectively. Hyperhydrosis is also the frequent cause, associated with (5.0\%) patients. Disfigurement $117(97.5 \%)$ and discomfort 89 (74.2\%) were more common chief complaints of the patients. The mean duration of disease was $20.4 \pm 15.4$ months with ranged from 2 to 72 months. Majority of the patients 63(52\%) had duration of 1-12 months. Nail fold changes are associated with $37(30.8 \%)$ patients. More than a half $63(52.5 \%)$ of the patients had wet works, family history of onychomycosis (19.2\%), others personal history varied from 5 to $20.0 \%$ showed in the table.

Regarding the history of past illness and comorbidity it was observed that previous onychmycosis found 26(21.7\%), nail trauma 26(21.7\%) and immune suppression 6(5.0\%). In endocrinopathies, hypothyroidism was observed in 1(0.8\%), DM 6(5.0\%) and others 26(21.7\%). Regarding the pattern of nail change thickening of nail plate 88(73.3\%), onycholysis 
67(55.8\%), subungunal hyperkeratosis 61(50.8\%), roughening of nail plate 42 (35\%), yellowish discoloration 38(31.75) and brownish-yellow discoloration 29(24.2\%) were more common changes. Paronychia was observed in 34 (28.3\%)cases. In concomitant fungal infection, it was observed that T. manum in $6(5.0 \%)$, T. pedis $3(2.5 \%)$, Interdigital intertrigo $2(1.7 \%)$ and T. cruris $1(0.8 \%)$. Site of nail involvement it was observed that right hand 27(22.5\%), left hand 18(15.0\%) and both hand 30 (25.0\%). Most 35(29.2\%) of the patients had single finger nails involvement. In case of toe nails it was observed that right foot was 22(18.3\%), left foot 15(12.5\%) and both foot 42 (35.0\%). Majority (22.5\%) of the patients had single toe nails 27(22.5\%) involvement.

\section{Table-I}

\begin{tabular}{lcc}
\multicolumn{2}{c}{$\begin{array}{c}\text { Distribution of the study patients according to } \\
\text { particulars of the patients }(n=120)\end{array}$} \\
$\begin{array}{lcc}\text { Number of } \\
\text { Particulars of } \\
\text { the patients }\end{array}$ & Percentage \\
\hline Age & & \\
$<18$ & 6 & 5.0 \\
$21-30$ & 61 & 50.8 \\
$31-40$ & 21 & 17.5 \\
$41-50$ & 18 & 15.0 \\
$51-60$ & 10 & 8.3 \\
$>60$ & 4 & 3.3 \\
Mean \pm SD & 32.8 & \pm 14.0 \\
Sex & & \\
Male & 64 & 53.3 \\
Female & 56 & 45.8 \\
Occupation & & \\
Service & 25 & 20.8 \\
Business & 14 & 11.7 \\
Student & 33 & 27.5 \\
Housewife & 36 & 30.0 \\
Farmer & 2 & 1.7 \\
Miscellaneous & 10 & 8.3 \\
Economic Status & & \\
Low income & 11 & 9.2 \\
Low-middle class group & 72 & 60.0 \\
Upper-middle class group & 37 & 30.8 \\
Mean \pm SD & 14241 & \pm 9642 \\
\hline
\end{tabular}

National children policy of Bangladesh(Bangladesh Gezette,2002) as well as UNICEF defined child as any person under 18 years of age

Low income: $<5,000$ per month; High income $>60,000$ Low-middle class group: 5,000-<20,000; Upper-middle class group: 20,000-<60,000
Table-II

\begin{tabular}{lcc}
\multicolumn{2}{c}{$\begin{array}{c}\text { Distribution of the study patients according to } \\
\text { chief complaints }(n=120)\end{array}$} \\
$\begin{array}{lcc}\text { Number of } \\
\text { Chatients }\end{array}$ & Percentage \\
\hline $\begin{array}{l}\text { Disfigurement } \\
\text { Discomfort }\end{array}$ & 117 & 97.5 \\
Pain & 89 & 74.2 \\
Associated changes in nail folds & 39 & 24.2 \\
Duration of the disease & 37 & 30.8 \\
1 -12 months & 63 & 52.5 \\
$13-24$ months & 25 & 20.8 \\
$>24$ months & 32 & 26.7 \\
Personal history & & \\
Wet works & 63 & 52.5 \\
Family history of & 23 & 19.2 \\
onychomycosis & & \\
Wearing closed footwear & 19 & 15.8 \\
for long time & & \\
Sharing of common facilities & 18 & 15.0 \\
Animal handling & 16 & 13.3 \\
Hyperhydrosis & 6 & 5.0 \\
\hline
\end{tabular}

\section{Table-III}

Distribution of the study patients according to history of past illness and comorbidity $(n=120)$

\begin{tabular}{lcc}
$\begin{array}{l}\text { History of past illness } \\
\text { \& comorbidity }\end{array}$ & $\begin{array}{c}\text { Number of } \\
\text { patients }\end{array}$ & Percentage \\
\hline Previous onychmycosis & 26 & 21.7 \\
Nail trauma & 26 & 21.7 \\
Febrile illness & 0 & 0.0 \\
Endocrinopathies & & \\
Hypothyroidism & 1 & 0.8 \\
Diabetes Mellitus & 6 & 5.0 \\
Addison's disease & 0 & 0.0 \\
Hyperthyroidism & 0 & 0.0 \\
Hypoparathyroidism & 0 & 0.0 \\
Chronic mucocutaneous & 0 & 0.0 \\
candidiasis & & \\
Cushing's syndrome & 0 & 0.0 \\
Others & & \\
Immune suppression & 6 & 5.0 \\
Peripheral vascular disease & 0 & 0.0 \\
HIV disease & 0 & 0.0 \\
Drug history & 0 & 0.0 \\
\hline
\end{tabular}


Table-IV

\begin{tabular}{|c|c|c|}
\hline \multicolumn{3}{|c|}{$\begin{array}{l}\text { Distribution of the study patients according to } \\
\text { clinical examination }(n=120)\end{array}$} \\
\hline Clinical examination & $\begin{array}{l}\text { Number of } \\
\text { patients }\end{array}$ & Percentage \\
\hline \multicolumn{3}{|l|}{ Pattern of nail change } \\
\hline Thickening of nail plate & 88 & 73.3 \\
\hline Onycholysis & 67 & 55.8 \\
\hline Subungunal hyperkeratosis & 61 & 50.8 \\
\hline Roughening of nail plate & 42 & 35.0 \\
\hline Yellowish discoloration & 38 & 31.7 \\
\hline Brownish-yellow discoloration & 29 & 24.2 \\
\hline Blackish discoloration & 10 & 8.3 \\
\hline Whitish discoloration & 9 & 7.5 \\
\hline Destruction of nail plate & 7 & 5.8 \\
\hline Chalky white spot & 2 & 1.7 \\
\hline Pitting & 2 & 1.7 \\
\hline Paronychia & 34 & 28.3 \\
\hline \multicolumn{3}{|l|}{ Concomitant fungal infection } \\
\hline T. manuum & 6 & 5.0 \\
\hline T. pedis & 3 & 2.5 \\
\hline Chronic paronychia & 0 & 0.0 \\
\hline Interdigital intertrigo & 2 & 1.7 \\
\hline T. corporis & 0 & 0.0 \\
\hline T. capitis & 0 & 0.0 \\
\hline T. cruris & 1 & 0.8 \\
\hline
\end{tabular}

\section{Discussion:}

In a study by Grag et al. comprised 90 patients with onychomycosis showed that the male: female ratio was $3: 1$ and the mean age was $29.40 \pm 13.61$ years. Office workers and students constituted the majority(63.3\%) of patients. 35.5\% of the patients who presented with disease of less than 6 months and $70.8 \%$ of the patients who had presented with the disease duration of more than 6 months, had multiple nail involvement. Disease was associated with history of trauma in $14.14 \%$ of patients. Fingernails were involved in $60 \%$, toenails in $13.34 \%$. The most common clinical features was discoloration, which was observed in $100 \%$ of the patients(brown-black in 40\%, yellow-brown in32\%, grayish-black in $20 \%$ and white or grayish-white in 8\%).Other signs included subungual hyperkeratosis(48\%), onycholysis(37\%) and paronychia(12\%). ${ }^{6}$

Table-V

Distribution of the study patients according to
site of nail involvement $(n=120)$

Site of nail involvement

Number of Percentage patients

\section{Finger nails}

Right hand

Left hand

27

22.5

Both hand

$18 \quad 15.0$

No

$30 \quad 25.0$

45

37.5

Number of finger nails

1

2

3-8

9-10

35

29.2

$\begin{array}{ll}7 & 5.8\end{array}$

$32 \quad 26.7$

$\begin{array}{ll}0 & 0.0\end{array}$

Toe nails

Right foot

22

18.3

Left foot

12.5

Both foot

15

35.0

No

41

34.2

Number of toe nails

\begin{tabular}{lcc}
1 & 27 & 22.5 \\
2 & 26 & 21.7 \\
$3-8$ & 24 & 20.0 \\
$9-10$ & 1 & 0.8 \\
\hline
\end{tabular}

In one study was by Das et al. with 85 cases, 44 cases showed the presence of fungus (either by $\mathrm{KOH}$ preparation and / or fungal culture) amounting to $51.76 \%$ positivity. Among the 44cases, the mean age was 41.41 14.64 years (range: 15 -65years) with a male to female ratio was 1.1 . The patient mostly belonged to upper middle class (59\%) background followed by lower middle class (36.36\%) and poor (4\%) section of population. Occupation (especially wet work) was not found to have a significant association with their study population. Among those 44 cases, the infecting fungal agents were predominantly dermatophytes 22(50\%) cases and the rest were due to yeast $12(27.27 \%)$ cases and moulds $10(22.72 \%)$ cases. ${ }^{7}$

Gupta et al. showed that among 130 patients, male: female was 98:32 and they were between 8-76 years of age. (mean $41.35 \pm 14.98$ years). The prevalence of onychomycosis was higher among farmers and office 
workers (20\% each). Finger or toe nails were exclusively involved in 56.9 and $32.3 \%$ respectively while there were involved concurrently in the rest of the $10.8 \%$ patients. Ten(7.69\%) patients had associated peripheral vascular disease,18(13.8\%) patients had history of trauma. Animal handling in 79(60.78\%) and family history of onychomycosis were reported by 34(26.15\%) patient. The predominant nail changes observed were discoloration in 120 patients(92.3\%), subungual hyperkeratosis in 89(68.5\%), onycholysis in 35(26.9\%), dystrophy in 49(37.7\%), leukonychia in 19(14.6\%) and paronychia in 14(10.7\%) patients respectively. Pitting, beau's lines and melanonychia were some of the other nail changes observed. ${ }^{3}$

In one study by Sayed et al. among 772 patients (520 women, 252 men; mean age 40.3 years) direct microscopy was positive in 256 cases (33.2\%). Of this 230 were correlated with positive cultures and 26 with negative culture. However, cultures were positive in 419 patients\% (54.3\%; 260 women and 159 men). Among male patient, cultures was positive in $63.1 \%$ of cases, where half of the female patients who presented for consultation had a positive result $(\mathrm{p}<0.05)$. Three children were suspected to have onychomycosis, but in all of them cultures were negative. The ratio of fingernail / toenail onychomycosis was 1.9. Fingernail involvement was predominant in females(76.2) whereas toenail involvement was predominant in males(53.6\%). ${ }^{8}$

In an observational study by Bokhari et al. of 100 patients seventy two women (mean age, $32.6 \pm 14.8$ years) and twenty eight men (mean age $40.6 \pm 15.8$ years) were studied. Females outnumbered males (2.6:1, $\mathrm{p}<0.001)$. Of the 72 female patients, the number of housewives or housemaids was statistically significant $(\mathrm{p}<0.001)$. Fingernails were involved in 50 patients, toenails in 23 and both fingernails and toe nails in 27 patients. The number of nails involved ranged from 1 to 20. Six patients showed involvement of all 20 nails. The most common clinical feature was discoloration seen in $100 \%$ of patients. (brown-black in $40 \%$, yellow-brown in $32 \%$, grayish-white in $8 \%$ ). Other signs included onycholysis,(37\%), paronychia(34\%), subungual hyperkeratosis (23\%), broken nails(18\%), pitting(15\%),ridging(9\%), leukonychia(8\%), beau's lines(7\%) and onychogryphosis(3\%). Onychomycosis is more common in women of 20-40 years of age. Distolateral subungual onychomycosis and candidal onychomycosis are the most common clinical presentation. ${ }^{9}$

In one study, by Kaur et al. onychomycosis was seen affect all ages ranging from 5 years to 67 years, the mean age being 31.72 years and the majority of the cases were males. As many as 51 (85\%) patients were living in urban areas, while only 9 (15\%) came from rural areas. One patient (1.67\%) was in professional group and others were more or less equally distributed in other groups (i.e., housewife, agriculture, laborer, industrial worker, electrical, students and others). Most patients were involved in domestic activities (33.33\%), the most common being cooking followed by stitching and tailoring. Among clinical features, there was history of trauma preceding nail involvement in $3(5 \%)$ patients. One patient (1.67\%) gave history of onychophagia. Family history of fungal infection of nails could be elicited in 4 (6.67\%) of 60 patients. History of contact with the cattle and pets was presents in 11 (18.67\%) patients. It was observed that patients who were in habits of wearing chappals had much less incidence of toenail involvement (2/29) as compared to patients used to wearing shoes and socks (14/20), shoes only $4 / 5)$ or bare foot (4/6). Onychomycosis was limited to only one nail in 10/60 (16.67\%) cases, while 50 patients (83.33\%) showed involvement of two or more nails. The most common findings seen on examination of nails were subungual hyperkeratosis, Presence of coexisting fungal infection in other parts of the body was noted in 27(45\%) patients, the most being Tinea manuum. Other skin / systemic disorders were present in 23 (38.33\%) patients, hyperhydrosis being the most frequent. Rate of fungus was higher in nail samples collected by drilling (83.33\%) than scraping (66.67\%). ${ }^{10}$

Adhikari et al. conflicted a cross sectional study, which involve thirty four clinically suspected cases of onychomycosis. Among them there were 18 males (52.94\%) and 16 females (47.06\%), the male to female ratio being 1.125:1. Young adult in age group of 21-30 years were mainly affected. Fingernails were involved in 14 cases $(41.18 \%$ ) and toe nails were involved in 20 cases (58.82\%). Both finger and toenail involvements were not noticed in the same patient. Big toe nail involvement was the most common toe nail involved and it was seen in 11 cases (55\%). ${ }^{11}$ 
A study was undertaken in 488 patients suspected of onychomycosis by Chadeganipour et al. The study population comprised 310 (635\%) females and 178 (36.5\%) males, ranging in age from 1 to 80 years (mean age 42 year). Onychomycosis was found to be the commonest in housewives, followed by laborers working in petroleum industries and office workers. Prolonged moisture was the major predisposing factor, followed by occlusive footwear. Finger nail onychomycosis is recognized in 141 (72.7\%) and toenail onychomycosis in 53 (27.3\%) cases. Simultaneous involvement of both finger and toenails were not seen. The prevalent clinical form of onychomycosis in adults was distal followed by proximal subungual hyperkeratosis and in children under 7 years of age, candidal paronychia was the commonest clinical pattern. ${ }^{12}$

The majority of the patients in our study were between the ages of 21 to 30 years (44.2\%). This attributed to the fact that onychomycosis may be considered a cosmetic problem rather than disease process in this region and so it was younger patient, who are more conscious of their appearance, who came forward for therapy. There were only six children highlighting the fact that this disease is less common in this age group. The low prevalence in children may be attributed to a difference in nail plate structure, a lack of cumulative trauma and increased growth rate of nails with subsequent elimination of the fungus.

Various studies had showed no sex difference. In our study males are affected slightly more than the female (1.1:1). The increased prevalence of onychomycosis in men has been suggested to be the result of more trauma to nails and the more common use of occlusive footwear. Most of the patients were house wife (30.0\%), followed by students (27.5\%), service holder (20.8\%). Household wet works appears to be an important predisposing factor in housewives.

Range of duration of the disease was between 2 to 72 months. Majority of them had the disease of less than 12 months more than a half (52.5\%) of the patients had involved in wet works, e.g. kitchen work, washing of cloths. Family history of any onychomycosis sharing of common facilities present in $18.8 \%$ \& $15.0 \%$ of patient respectively. Hyperhydrosis is also the frequent cause, associated with (5.0\%) patients. Association of hyperhydrosis was also found in other study. ${ }^{10}$ Occlusion, warmth and moisture provided by occlusive footwear predispose to onychomycosis was reported (15.8\%). In this study history of previous onychomycosis (21.7\%) and nail trauma (21.7\%) were found in significant number of patient. Onychomycosis that occur in farmer $(1.7 \%)$ could be explained by their occupational predisposition to trauma, $5.0 \%$ patient were diabetic. Clinical diagnosis of onychomycosis is based on the basis of various changes, in the affected nails. In this study, among the pattern of nail changes thickening of nail plate, onycholysis, subungual hyperkeratosis, roughening of nail plate and yellowish discoloration were common.

Presence of coexisting fungal infection in other parts of the body was noted in 120 patients. Among them T. manuum was common followed by T. pedis, interdigital intertrigo and T. cruris. Fingernails were more commonly involved than toenails. Most of the patient had single fingernail (29.2\%) and single toenail (29.2\%) involvement. Another study had shown more toenails involvement than fingernails. ${ }^{13}$

\section{Conclusion:}

This was a study on a limited number of cases. Future studies must include economical support, then large sample size could be ensured and study finding would be more reliable. There is a great need of epidemiological studies also, with sufficient follow-up, systematic reviews and meta-analyses on this issue.

\section{References:}

1. Verma S \& Hefferman MP. 'Fungal diseases: onychomycosis'. K Wolf, LA Goldsmith, S Katz, BA Gilchrest, AS Paller, DJ Leffell (eds). Fitzpatrick's Dermatology in General Medicine. 7th edn. USA:McGraw-Hall Companies; 2008.pp. 1817-18.

2. James WD, Berger TG \& Elston DM. 'Diseases resulting from fungi, yeast :onychomycosis' Andrews' Diseases Of Skin Clinical Dermatology. 10th edn, Philadelphia, USA :Elsevier ; 2006. pp. 305-6.

3. Gupta M, Sharma NL, Kanga AK, Mahajan VK \& Tegta GR. 'Onychomycosis : Clinicomycologic study of 130 patients from Himachal Pradesh, India’. Indian Journal of Dermatology venerology \& leprology2007; 73(6): pp. 389-92.

4. Ilkit M. 'Onychomycosis in Adana, Turkey : A 5 year study'. International Journal of Dermatology 2005; 44(10): pp.851-4. 
5. Ikram A, Hussain W, Khurshid U, Butt T \& Wiqar MA. 'Pattern of Mycological infections in our set up'. Pakistan Journal of Pathology2007; vol. 18(3): pp. 95-8.

6. Grag A, Venkatesh V, Singh M, Pathak KP, Kaushal GP \& Agrawal SK. 'Onychomycosis in central India: A clinicoetiologic correlation'. International Journal of Dermatology2004; vol. 43: pp. 498-502.

7. Das NK, Ghosh P, Das S, Bhattacharya S, Dutta RN \& Sengupta SR. 'A Study on the etiological agent and clinicomycological correlation of finger nail onychomycosis in Eastern India'. Indian Journal of Dermatology2008; vol. 53(2): pp. 75-9.

8. Sayed FE, Ammoury A, Haybe RF \& Dhaybi R. 'Onychomycosis in Lebanon: A Mycological survey of 772 patients'. Mycosis 2006; 49(3): pp. 216-9.

9. Bokhari MA, Hossain I, Jahangir M, Haroon TS, Aman S \& Hurshid K. 'Onychomycosis in Lahore, Pakistan'.
International Journal of Dermatology1999;vol. 38: pp. 591-5.

10. Kaur R, Kashyap B \& Makkar R. 'Evaluation of clinicomycological aspects of onychomycosis'. Indian Journal of Dermatology2008; 53(4): pp. 174-8. g

11. Adhikari L, Gupta AD, Pal R \& Singh TSK. 'Clinico-etiologic correlates of Onychomycosis in Sikkim'. Indian Journal of Pathology \& Microbiology2009; 52(2): pp. 194-7.

12. Chadeganipour M, Nilipour S \& Ahmadi G. 'Study of Onychomycosis in Ispahan, Iran’. Mycosis2008; vol. 52(2): pp. 153-7.

13. Asadi MA, Dehghani R \& Sharif MR. 'Epidemiologic study of Onychomycosis and Tinea Pedis in Kashan, Iran'. Jundishapur Journal of Microbiol2009; 2(2): pp. 61-4.

14. Chi CC, Wang SH \& Chou MC. 'The causative pathogens of onychomycosis in southern Taiwan'. Mycosis2005; 48(6): pp. 413-20. 